\title{
Drei ereignisreiche Monate in New York City - Wahlstation bei einer amerikanischen Anwaltskanzlei
}

\author{
Dr. Susanne Fischer \\ Mitglied im Bundesvorstand, dort zuständig für Mitglieder in \\ Ausbildung; Rechtsanwältin, Düsseldorf
}

Während des Referendariats bot sich mir die Gelegenheit, meine Wahlstation in den Sommermonaten Juli bis September in einer US-amerikanischen Anwaltskanzlei in New York City abzuleisten. Durch meine Anwaltsstation in einem deutschen Unternehmen wurde ich auf die Kanzlei Gibbons P.C. aufmerksam gemacht. Nach der Bewerbung für das dortige Foreign Legal Internship Program (FLIP), das insbesondere deutsche Referendar(inn)e(n) anspricht, hatte ich rasch die Zusage und dem Aufenthalt in der amerikanischen Metropole stand nichts mehr entgegen.

\section{Wohnen in Manhattan}

Nach ein wenig Recherche im Internet und mehreren Gesprächen mit vorherigen Referendaren hinsichtlich des Wohnens in New York stand ich vor der Entscheidung, ob ich die Zeit in einem Wohnheim, in einer WG mit Mitbewohnern aus verschiedenen Ländern oder in einem eigenen Apartment leben möchte.

Von Beginn an wusste ich jedoch, dass ich trotz der horrenden Mietpreise zentral in Manhattan wohnen wollte, um das Leben in der pulsierenden Stadt live mitzuerleben, zudem wurden mir dadurch alle Aktivitäten und Unternehmungen extrem erleichtert.

Alle genannten Varianten des Wohnens in der Stadt, die niemals schläft, haben Vor- wie Nachteile, insbesondere die Wohnungssuche von Deutschland aus gestaltete sich nicht immer als einfach.

So habe ich mich dann nach einer gründlichen Abwägung dafür entschieden, zunächst in das Webster zu ziehen, ein Wohnheim nur für Frauen. Diese in Midtown Manhattan zentral gelegene Unterkunft kann ich uneingeschränkt weiterempfehlen. Ich habe unheimlich schnell viele sympathische Frauen kennengelernt und trotz des Umstands, dass wir doch weitgehend unter Deutschen geblieben sind, was der Erweiterung der Sprachkenntnisse nicht unbedingt zuträglich war, habe ich zahlreiche Kontakte knüpfen können und wir haben alle gemeinsam viel auf die Beine gestellt. Zudem ist das Webster für New Yorker Verhältnisse mit einem Preis von derzeit ca. \$ 265,- pro Woche (im Sommer 2010 umgerechnet etwa 210,- Euro) inklusive Frühstück und Abendessen wirklich günstig, wenn man sich demgegenüber die horrenden Preise für Wohnen und Essen dort vor Augen führt.

Das Wohnheim erinnert sehr an ein Internat, man hat ein kleines, sehr gepflegtes Einzelzimmer und teilt mit den anderen Frauen auf der jeweiligen Etage die Duschen und die Toiletten. Die Sorge mancher Kollegin, morgens lange Schlangen vor den Duschen anzutreffen, war unbegründet, die Benutzung gestaltete sich als absolut unproblematisch.

Neben einem Speisesaal und einem wunderbaren Garten, den man jederzeit nutzen kann, gibt es mehrere Gemeinschaftsräume und eine grandiose Dachterrasse mit Blick auf Manhattan, auf der wir viele gemeinsame Abende verbracht und den Ausblick, insbesondere auf das nur wenige Blocks entfernte Empire State Building, genossen haben.

Im dritten und letzten Monat meines Aufenthalts bin ich dann aus verschiedenen Gründen vom Webster in ein eigenes Apartment gezogen, was ich vor Ort in einer nicht wenig aufreibenden Suche schlussendlich auf der Upper West Side gefunden habe. Auf diese Weise habe ich New York noch einmal von einer anderen Seite kennengelernt, denn in der Wohnung habe ich mich im Gegensatz zum Wohnheim um den Einkauf, das Kochen und das Putzen selbst kümmern müssen.

\section{Das Visum}

Zeitaufwändig und mit allerlei Bürokratie verbunden war die Erlangung des erforderlichen Visums der Kategorie „J1“. In meinem Fall übernahm es meine Ausbildungskanzlei, die German American Chamber of Commerce (GACC) in New York als Sponsororganisation zu gewinnen, was ein erforderlicher Schritt zum erfolgreichen Erhalt des Visums darstellt. Nach dem Zusammentragen aller möglichen Unterlagen und der Überweisung einer stattlichen Summe erhielt ich dann das erforderliche Dokument, mit dem ich beim Konsulat in Frankfurt mein Visum beantragen konnte.

Nach der Bezahlung weiterer Gebühren habe ich das notwendige Visumsinterview bei der Konsularabteilung in Frankfurt am Main vereinbart und drei Wochen vor meiner Abreise auch erfolgreich absolviert. Mein Reisepass mit dem Visum wurde mir daran anschließend und rechtzeitig vor dem Abflug zugeschickt.

\section{Die Ankunft und die Arbeit}

Nach meiner Ankunft hatte ich zunächst einige Tage Zeit, mich zu akklimatisieren und mir einen ersten Eindruck von der Stadt zu verschaffen. Dabei habe ich versucht, Manhattan weitgehend zu Fuß zu erschließen, soweit dies das Wetter - New York liegt auf demselben Breitengrad wie Neapel und war dementsprechend sehr heiß - und mein körperlicher Zustand unmittelbar nach den Examensklausuren erlaubten.

In der auf meine Ankunft folgenden Woche begann die Arbeit bei Gibbons P. C., einer Full-Service-Kanzlei mit insgesamt etwa 220 Anwälten in fünf Städten, was für amerikanische Verhältnisse als eher kleines Anwaltsbüro zu bezeichnen ist. Im New Yorker Büro arbeiten insgesamt 60 Anwälte. Die Kanzlei ist nur wenige Schritte vom Webster entfernt und zen- 
tral in Midtown Manhattan, direkt über der Penn Station, einem der Hauptbahnhöfe New Yorks, gelegen. Neben mir waren noch zwei weitere Referendare, eine Kollegin aus Hamburg und ein Kollege aus München, für ihre Wahlstation vor Ort, untergebracht waren wir in einem gemeinsamen Büro.

Nach der Begrüßung wurden wir gleich im ersten Gespräch von einem der uns zugewiesenen Partner nach unseren Interessengebieten befragt, dieser bemühte sich während unseres Aufenthalts immer wieder, Arbeiten aus diesen Bereichen an uns zu übertragen. Meine Aufgaben erledigte ich überwiegend in den Bereichen Corporate und Kartellrecht.

Die Referendarskollegin aus Hamburg, die unter anderem die amerikanische Staatsbürgerschaft besitzt und einen LL.M. in den USA erworben hat, arbeitete während der drei Monate dauerhaft für einen amerikanischen Anwalt im Bereich Arbitration, ein von ihr schon in Deutschland im Referendariat gewählter Schwerpunkt.

Die Arbeitszeiten für Referendare waren in der Regel montags bis freitags von 9 bis 17 Uhr. Die Betreuung war vorbildlich. Die deutsche Anwältin, die das oben genannte Programm betreut, nahm sich sehr viel Zeit und stand uns stets bei Problemen mit Rat und Tat zur Seite. Auch die anderen Anwälte lernten wir im Laufe unserer Stage kennen, regelmäßig ergab sich auf dem Flur oder in der Küche ein Gespräch, für Rückfragen standen die Türen jederzeit offen.

Die Arbeit war vielfältig. In den überwiegenden Fällen wurden mein Kollege und ich in Mandate mit Bezug nach Deutschland einbezogen, wobei wir keinesfalls ausschließlich Dolmetscherdienste erbringen, sondern auch Stellungnahmen zu bestimmten Themen auf Englisch schreiben mussten. Darüber hinaus habe ich mehrere Artikel zum europäischen und amerikanischen Kartellrecht in englischer Sprache verfasst.

Dies stellte durchaus eine Herausforderung für mich dar, denn es ist eine Sache, die Sprache zu verstehen und sich in ihr zu verständigen, eine ganz andere jedoch, auch wissenschaftliche Texte darin zu verfassen. Schließlich gelangen sämtliche Arbeiten doch, nicht zuletzt auch dank der Hilfe meiner Kollegen und diverser elektronischer Wörterbücher.

Über die tägliche Arbeit hinaus waren sowohl die Kanzlei wie die Ausbilder stets bemüht, uns den Aufenthalt so interessant und abwechslungsreich wie möglich zu gestalten. Wir haben verschiedenen Zeugenanhörungen ebenso beiwohnen dürfen wie Gerichtsverhandlungen, konnten beim New York Police Department (NYPD) an einem dort eigens eingerichteten Court Disziplinarverfahren gegen Polizisten verfolgen und schließlich sogar eine Polizeifahrt mit dem NYPD in der Bronx machen. Darüberhinaus besuchten wir das Büro der Kanzlei in Philadelphia, verbunden mit einem dort anhängigen Schiedsverfahren und einer anschließenden Stadtbesichtigung, bei der einer der dortigen Partner es sich nicht nehmen ließ, uns persönlich zu begleiten und uns das legendäre Philly Cheese Steak näher zu bringen.

Auch der Sport durfte nicht zu kurz kommen. Anlässlich des stattfindenden Fußballweltmeisterschaftsspiels Deutschland - Spanien organisierte ein sportbegeisterter Anwalt kur- zerhand am Nachmittag einen Tisch in einer Sports-Bar und lud uns deutschen Referendare zu Bier und Pizza ein.

Interessant war schließlich unsere regelmäßige Teilnahme am traditionellen Referendarsfrühstück in der Kanzlei Alston \& Bird, das freitags zwischen 8.00 und 9.30 Uhr stattfindet.

Neben dem Umstand, dass man hier auf eine Vielzahl weiterer deutscher Kollegen trifft, lernt man auch das Rechtssystem der USA besser kennen, denn bei jedem Frühstück trägt ein anderer Anwalt der Kanzlei in lockerem Rahmen zu verschiedenen Themen des amerikanischen Rechts vor.

\section{Leben in Manhattan}

Die täglichen Arbeitszeiten ließen mir genügend Zeit, Manhattan, New York und das nähere Umfeld mit all seinen Sehenswürdigkeiten zu erkunden.

Neben den typischen Orten, die man sich keinesfalls entgehen lassen sollte, wenn man zu Gast in dieser Metropole ist, habe ich sämtliche Stadtviertel Manhattans akribisch unter die Lupe genommen. Angesichts der Vielfältigkeit, die man dort zu sehen bekommt, fällt es schwer, einzelne Orte hervorzuheben, denn jeder Bezirk hat seinen ganz eigenen Reiz.

Häufiger aufgesucht habe ich jedoch Greenwich Village, SoHo sowie Chelsea. Speziell letzteres Viertel ist mit seinen zahlreichen Galerien ein Muss für jeden, der sich für Kunst interessiert.

Zum Ausruhen, Verweilen und Beobachten des stets regen Treibens laden die Grünanlagen des Battery Parks und solche rund um die NYU sowie das Flatiron Building ein.

Auch kulturell hat unsere Gruppe, die sich dort kennengelernt hat und zu überwiegenden Teilen aus Rechtsreferendar(inn)en bestand, einiges auf die Beine gestellt. Neben den diversen Museumsbesuchen ist besonders unser Opernbesuch in der Metropolitan Opera hervorzuheben. Kurz vor Beginn der Sommerpause kamen wir in den Genuss, dort das Ballett „Romeo und Julia“ sehen zu können und waren uns alle einig, mit dieser Aufführung etwas ganz Besonderes erlebt zu haben.

Schlussendlich musste natürlich auch das Nachtleben von uns eingehend inspiziert werden, auch hier haben wir trotz regelmäßigster Ausflüge in selbiges nur einen Bruchteil der zahlreichen Kneipen, Bars und Clubs kennenlernen können.

Auch der Besuch einer sogenannten „Hidden Bar“ stand in diesem Zusammenhang auf dem Programm. Eine Referendarskollegin, die diesen Abend organisiert hat, hat auf diese Weise der Liste unvergesslicher „New York-Erlebnisse“ einen weiteren Punkt hinzugefügt. Bei diesen Bars, auch als „Speakeasy“ bezeichnet, handelt es sich um geheime Bars, in denen zu Zeiten der Alkoholprohibition in den USA illegal Bier sowie hochprozentiger Alkohol ausgeschenkt wurde.

Von außen handelte es sich bei dem von uns besuchten Ort um eine ganz normale Hot-Dog-Bude. Um in den versteckten Teil zu kommen, mussten wir eine in dem Schnellimbiss gelegene Telefonzelle ansteuern und den Hörer abheben in der Hoffnung, dass sich am anderen Ende jemand meldet. Nach 
Nennung des bei der Reservierung erhaltenen Codewortes öffnete sich eine nicht ohne weiteres sichtbare Tür in einer weiteren Wand und gab uns den Weg in die Bar frei.

Erholung von der Stadt und den zahlreichen Streifzügen durch die New Yorker Nächte findet sich in den Sommermonaten an den Stränden von Long Island, nach einer knapp einstündigen Zugfahrt kann man dort wunderbar entspannen und muss sich angesichts der Ruhe und der Weitläufigkeit dort immer wieder vor Augen führen, dass die Weltstadt nur einen Katzensprung entfernt ist. Natürlich laden auch die Hamptons dazu ein, den Lärm der Stadt bei einer Fahrradtour mit anschließendem Bad im Ozean hinter sich zu lassen.

Wer einmal etwas weiter hinaus möchte aus New York, der kann beispielsweise Städte wie Washington D. C. oder Boston besuchen. Diverse Busunternehmen bieten beinahe stündlich Reisen dorthin an und nach etwa vierstündiger Fahrt ist die Hauptstadt der Vereinigten Staaten bzw. von Massachusetts erreicht.

Die Ausführungen über das Leben und meine Erlebnisse in Manhattan ließen sich mannigfach erweitern. Zusammenfassend kann ich jedenfalls festhalten:

Ich habe den gesamten Aufenthalt meiner Wahlstation in New York City keinen einzigen Tag bereut. Den Sommer in dieser Stadt so unmittelbar nach dem schriftlichen Examen verbringen zu können, war eine einmalige Erfahrung, die ich sicherlich nie im Leben vergessen werde.

\section{Veranstaltungshinweise}

\section{Feministischer Juristinnentag}

\section{6.-8. Mai 2011 in der FH Frankfurt a.M.}

Veranstalterin ist der Verein „Frauen streiten für ihr Recht e.V.“, Frankfurt a.M. In der feministischen Rechtszeitschrift STREIT werden jeweils die Resolutionen und Programme, wie auch einzelne Vorträge publiziert.

Im Bereich des Familienrechts soll es um das gemeinsame Sorgerecht sowie die Gesetzentwürfe zum Sorgerecht nicht mit der Mutter verheirateter Väter gehen. Darüber hinaus werden Fragen des Unterhaltsrechts und die Gestaltung von Eheverträgen aufgegriffen. Ein arbeitsrechtlicher Schwerpunkt wird sich mit dem Thema Arbeitsmarkt, Wirtschaftskrise und ihre Auswirkungen auf Frauen befassen. Zusätzlich werden aktuelle Diskussionen (Mindestlohn, Arbeitszeit u.ä.) aufgegriffen. Einige Angebote richten sich speziell an Nebenklagevertreterinnen (Glaubhaftigkeitsgutachten, Fragen der Kooperation mit der psychosozialen Prozessbegleitung und Opferentschädigung). Daneben sollen aber auch „Täterinnen“ Thema sein. Eine weitere AG wird sich mit aktuellen aufenthaltsrechtlichen Themen befassen. Schließlich ist ein Forum zur inneren Sicherheit geplant, das Themen wie Terrorismus, häusliche Gewalt und Diskriminierung einschließt. Das Programm und weitere Informationen: http://www.feministischer-juristinnentag.de/index.html und in der STREIT.

\section{EWLA-Kongress}

\section{6.-27. Mai 2011 in Malta}

Der Kongress steht unter dem Motto „Empowering Women Lawyers across Europe through Networking in the Pursuit of Justice“. Sprechen wird u.a. Dr. Viviane Reding, Vizepräsidentin der Europäischen Kommission und Kommissarin für Justiz, Grundrechte und Bürgerschaft. Programm: http://www. ewla.org/.

\section{Anwältinnenkonferenz - DAT 2011}

\section{2.-4. Juni 2011 in Straßburg}

Die Arbeitsgemeinschaft Anwältinnen veranstaltet ihre 13. Anwältinnenkonferenz im Rahmen des Deutschen Anwaltstages in Straßburg. Die Themen am 2. Juni 2011, 14.00 bis 16.00 Uhr sind: „Sexualisierte Kriegsgewalt vor Internationalen Strafgerichten“ unter Mitwirkung von Dr. Monika Hauser (Gründerin und geschäftsführendes Vorstandsmitglied von medica mondiale e.V., Köln), Dr. Kathrin Greve, LL.M., Berlin, Claudia Hoefer (International Criminal Tribunal for the former Yugoslavia, Den Haag), Mag. Renate Winter (Richterin in der Berufungskammer des Spezialgerichtshofs von Sierra Leone (SCSL), Den Haag), Prof. Dr. Dr. h.c. Angelika Nußberger, M.A. (Richterin am Europäischen Gerichtshof für Menschenrechte, Straßburg), Mechtild Düsing (Rechtsanwältin, Münster).

Von 16.30 bis 18.00 Uhr geht es um „Europa ist weiblich. Anwältinnen in Deutschland, Frankreich und der Schweiz Anwältinnen an die Macht und in die Aufsichtsräte" unter Mitwirkung von Avivah Wittenberg-Cox (Autorin und Beraterin in Gender-Angelegenheiten, „WHY \& HOW Women Mean Business - A Walk around Womenomics“, Paris), Dr. Maria Wolleh (Rechtsanwältin, Partnerin von Mannheimer Swartling Advokatbyrå AB und Secretary for IBA's Women Lawyers' Interest Group, Berlin), Valérie Tandeau de Marsac (Rechtsanwältin, Partnerin von Jeantet Associés, Gründerin und Präsidentin von voxfemina, Paris), Ines Pöschl (Rechtsanwältin, Partnerin von Kellerhals Anwälte, Zürich), Dr. Barbara Mayer (Rechtsanwältin, Geschäftsführende Partnerin Friedrich Graf von Westphalen \& Partner, Freiburg).

Der Frühstücksempfang am 3. Juni 2011, 8.30-9.30 Uhr ist offen für Mitglieder, Interessentinnen und Interessenten. Moderation: Rechtsanwältin Dr. Eva-Dorothee Leinemann, Berlin.

Weitere Informationen: http://www.dav-anwaeltinnen.de/ und http://www.anwaltverein.de/DAT. 